\title{
HISTORIA \\ DE LA ENFERMERÍA CHILENA: UNA REVISIÓN DESDE LAS FUENTES $^{1}$
}

\author{
HISTORY \\ OF CHILEAN NURSING: A REVIEW FROM THE SOURCES \\ HISTÓRIA \\ DA ENFERMAGEM CHILENA: UMA REVISÃO DAS FONTES
}

\author{
Elizabeth Núñez Carrasco* \\ LyLIAN MACÍAS INZUNZA** \\ Rossana Navarro Torres*** \\ Simone De Souza Paiva****
}

\section{RESUMEN}

Objetivo: Identificar si en la producción de conocimiento de enfermería en Chile está como sujeto de estudio la historia de la enfermería chilena. Material y Método: Se realizó una búsqueda en fuentes secundarias que consideró las bases de datos Pro Quest, Scopus, Web of Science y Pubmed. Para la estrategia de búsqueda se utilizaron los descriptores "history", "nursing" y "Chile" unidos por el booleano "and", que arrojó 20 publicaciones. Se seleccionaron sólo aquellos que fueran publicaciones científicas y que tuvieran una relación directa con la Historia de la Enfermería en Chile. Además, se buscó libros y tesis de postgrado en los archivos de bibliotecas nacionales, utilizando los descriptores "historia", "enfermería" y "Chile", encontrándose un total de 5 manuscritos. Resultados: La enfermería y su historia en Chile todavía es una temática incipiente en el país y se ha desarrollado en las áreas de la comprensión de la identidad de las enfermeras, de sus vínculos con la institucionalidad de los sistemas sanitarios y educativos. Conclusiones: La historiografía de la enfermería chilena es claramente un espacio en construcción y el desafío actual es su construcción desde la disciplina.

Palabras clave: Historia de la Enfermería; Rol de la Enfermera; Investigación en Enfermería.

\footnotetext{
ABSTRACT

Objective: To identify whether in the production of the Chilean nursing knowledge, the history of Chilean nursing has been a study topic. Materials and Methods: A secondary sources search was carried out using the Pro

${ }^{1}$ Investigación financiada por la Universidad de Santiago de Chile. Dicyt 021702NC “Cuidados de Enfermería: una revisión historiográfica”.

*Doctora en Enfermería. Universidad de Santiago de Chile, Escuela de Enfermería, Santiago, Chile. Email: elizabeth.nunez@usach.cl Autor de correspondencia.

**Magíster en Enfermería. Universidad de Santiago de Chile, Escuela de Enfermería, Santiago, Chile. Email: lylian.macias@usach.cl

***Enfermera. Universidad de Santiago de Chile, Escuela de Enfermería. Santiago, Chile. Email: rossana.navarro@usach.cl

****Doctora en Enfermería. Universidad de Santiago de Chile, Escuela de Enfermería, Santiago, Chile. Email: simone.desousa@usach.cl
} 
Quest, Scopus, Web of Science and Pubmed databases. For the search of the articles, the descriptors, "history", "nursing" and "Chile" were used, resulting in 20 publications. Only those that were scientific publications and had a direct relationship with the History of Nursing in Chile were selected. In addition, books and postgraduate theses were searched in the archives of national libraries, using the descriptors "history", "nursing" and "Chile", which resulted in the search of 5 manuscripts. Results: Nursing and its history in Chile is still an emergent topic in the country and has been developed in the areas of understanding the identity of nurses, their links with the institutional framework of health and education systems. Conclusions: The historiography of Chilean nursing is clearly a space under construction and the current challenge is its construction from within the discipline.

Key words: History of Nursing; Nurse’s Role; Research in Nursing.

\section{RESUMO}

Objetivo: Identificar se na produção do conhecimento da enfermagem no Chile tem como objeto de estudo a história da enfermagem chilena. Material e Método: Realizou-se uma busca de fontes secundárias, para as quais foram consideradas as bases de dados Pro Quest, Scopus, Web of Science e Pubmed para a busca de artigos científicos, utilizando os descritores "history", "nursing" e "Chile", identificando 20 publicaçóes. Somente aqueles que eram publicaçôes científicas e que tinham relação direta com a História da Enfermagem no Chile foram selecionados. Além disso, livros e teses de pós-graduação foram pesquisados nos arquivos de bibliotecas nacionais, utilizando os descritores "história", "enfermagem" e "Chile", encontrando um total de 5 manuscritos. Resultados: A Enfermagem e sua história no Chile ainda é um tema incipiente no país e vem sendo desenvolvida nas áreas de compreensão da identidade do enfermeiro, suas vinculaçóes com a institucionalidade dos sistemas de saúde e educação. Conclusóes: A historiografia da enfermagem chilena é claramente um espaço em construção e o desafio atual é sua construção a partir da disciplina.

Palavras-chave: História da Enfermagem; Papel da enfermeira; Pesquisa em Enfermagem.

Fecha de recepción: 26/11/2017 Fecha de aceptación: 24/09/2018

\section{INTRODUCCIÓN}

La enfermería es una disciplina que durante siglos ha estado asociada a un oficio de mujeres y, por ende, su valoración social ha estado marcada por el avance de la mujer al interior de las sociedades. Velandia ${ }^{(1)}$ señala que tanto la práctica de enfermería como la formación del personal y su selección están ligados a la transformación histórica del proceso social de producción y, por ello, el papel que juega la mujer al interior de las sociedades de un país mostrará el avance de la enfermería, ya sea como profesión o como disciplina.

Enfermería como espacio disciplinario durante los últimos 70 años se ha caracterizado por diseñar teorías y modelos conceptuales, que buscan describirla y explicarla ${ }^{(2)}$. Es así que la producción de conocimiento a nivel internacional estuvo primariamente centrada en desarrollar un cuerpo de conocimientos propios que permitiera sostener una postura epistemológica al interior de la propia enfermería y fuera de ella ${ }^{(3)}$. Ahora bien, comprendiendo que la enfermería ha definido una posición en el concierto de la producción científica $^{(4)}$, surge una primera interrogante: ¿Cuál sería la utilidad de producir conocimiento desde lo histórico? La respuesta está en que la producción del conocimiento histórico es contextualizar un personaje, un grupo social y/o un hecho que un historiador ha escogido para ser interpretado ${ }^{(5)}$.

Siguiendo en la idea anterior, surge una segunda interrogante: ¿Cuál es la utilidad de estudiar la historia de la enfermería? Para Lewenson y Kron ${ }^{(6)}$ los aportes de la historia es el autoconocimiento, es la forma de cómo se estructuran las ideas, permitiendo imaginar nuevas ideas, valores $y$ 
experiencias, creando así cultura y disciplina. Según esto, podemos decir entonces que la historia, para enfermería, sería un catalizador que le permite a sus seguidores comprender su propio proceso como grupo profesional y disciplinario.

Como consecuencia de esta narrativa, es posible señalar que durante décadas las enfermeras han realizado un esfuerzo por identificarse con lo propio y, así mismo, distanciarse de la imagen de subordinación de la imagen médica. Del mismo modo, se desprende que la enfermería posee una fuerte vinculación con las dinámicas sociales de las mujeres, por ello, los cambios de la presencia de la mujer fuera del espacio doméstico hacia el escenario de lo público permite comprender la imagen de las mujeres que decidieron ser enfermeras.

Aprender de las memorias es un proceso constructivo y creativo de traer el pasado al presente, y no en un entendido de quedarse en ese recuerdo, sino deconstruirlo, llegar a sus cimientos y comprenderlos. La Asociación Americana de Historia de la Enfermería ${ }^{(7)}$ describe que las presiones sociales que han dado forma a la enfermería en el pasado persisten en el presente, pero seguramente con nuevas formas; por lo tanto, recrear el pasado sin duda permite dibujar una trayectoria que tendrá una interpretación de acuerdo al lente del observador.

Comprendiendo que Chile es una sociedad que ha vivenciado un proceso histórico con características propias, es necesario contextualizar que la enfermería chilena a comienzos del siglo XX poseía una formación de oficio. En el censo del año 1920 se indica que 1.619 personas eran enfermeros, 628 hombres y 991 mujeres, y de ellos, 22 hombres y 68 mujeres eran extranjeras ${ }^{(8)}$. La formación profesional se inicia de manera institucional al interior de la Universidad de Chile en 1906, por la necesidad de instruir científicamente solamente a mujeres laicas que atendieran las necesidades crecientes de cuidados en los recintos hospitalarios, puesto que los cuidados de enfermería eran realizados por religiosas y/o practicantes. Durante esta época, Cora Mayers $^{(9)}$ recalcaba que la enfermera no debía ser la improvisación entre la buena voluntad, la falta de conocimientos y la falta de compromiso ético con las personas enfermas, elementos todos que mostraban la disputa inicial que se dio en la gran mayoría de los países, entre el cuidado doméstico y los primeros momentos de la profesionalización de los cuidados de enfermería. En este proceso particular de profesionalización, también es importante conocer si fueron las propias mujeres, los usuarios o la institucionalidad quienes lo lideraron.

Por el contexto relatado y las interrogantes planteadas, el objetivo de esta investigación fue identificar si la enfermera y/o la enfermería chilena ha sido sujeto historiográfico en la producción chilena de conocimiento disciplinario.

\section{MATERIAL Y MÉTODO}

Se utilizó como referente metodológico la historiografía, la cual busca explicar los significados atribuidos por el historiador y el sujeto histórico ${ }^{(10)}$. De acuerdo a lo anterior, se realizó una búsqueda en fuentes secundarias en dos tiempos: primero se realizó una búsqueda en bases de datos y simultáneamente se ejecutó una búsqueda en catálogos de bibliotecas nacionales. Para la primera búsqueda fueron consideradas las base de datos Pro Quest, Scopus, Web of Science y Pubmed, con los descriptores "history", and "nursing" and "Chile", entre los años 1990 y 2017, que arrojó un total de 20 publicaciones. Luego se seleccionaron solamente artículos científicos, quedando finalmente 6 artículos para el análisis. La segunda búsqueda consideró los archivos de bibliotecas universitarias, tales como Universidad de Santiago, Universidad de Chile, Universidad Valparaíso, Pontificia Universidad Católica de Chile y Universidad de Concepción, utilizando los mismos descriptores en espańol y limitando la búsqueda a libros y tesis de postgrado. De esta segunda fuente se seleccionaron 5 documentos.

Posterior a la selección de artículos, tesis y libros se realizaron preguntas a las fuentes secundarias que permitieron situar si la producción de conocimiento estaba situada en la historiografía requerida, es decir, si existía un sujeto histórico, un tiempo y un espacio. Así, para la revisión se utilizaron las siguientes preguntas: ¿Cómo se describe a las enfermeras y/o la enfermería? ¿Por qué se escoge la temporalidad estudiada? ¿Para qué realizar un relato historiográfico de las enfermeras y/o enfermería en Chile? 


\section{RESULTADOS}

\section{Elementos historiográficos en la producción científica de artículos y tesis}

En el artículo de Ayala y Núñez ${ }^{(11)}$ escogen a la enfermería chilena como su sujeto de observación, por la necesidad de descubrir el desarrollo de la identidad de las enfermeras durante los ańos cincuenta y comienzos del decenio del año 2000. Los autores concluyen que estudiar la historia de la enfermería chilena permitió identificar que esta identidad no se desarrolló en un proceso lineal, sino que fueron más bien movimientos superpuestos que trascendieron a lo largo del tiempo, permitiendo dibujar la construcción de la identidad de la enfermería como una comunidad en Chile. Hechos como las reformas en salud pública, la búsqueda de reconocimiento de las mujeres con formación universitaria, el golpe de Estado de 1973 y la reformulación de la identidad de las enfermeras, a propósito de las reformas de educación y salud, permitieron descubrir una identidad propia de la enfermería chilena.

Asimismo, Núñez, Urra y Pavez ${ }^{(12)}$ investigaron a enfermeras como sujetos históricos de la mitad del siglo XX, con el fin de describir y conectar el relato histórico de la enfermera chilena con las narrativas individuales, obteniendo así un relato identitario de enfermería, antes de los años 70 del siglo XX en Chile. Del mismo modo, se sitúan en el espacio geográfico nacional, ya que era un escenario fundamental que les dio sustento a la investigación, por los cambios trascendentales que ocurrieron en el país, tanto en política, economía y vida social de la época. Es así que la identidad de las enfermeras no sólo está influenciada por su contexto historiográfico, sino que por las autodefiniciones o microrrealidades, como actores que interpretan simultáneamente su propia historia de vida, desde la proyección del futuro y del contexto de acción de donde se encuentran.

Por otra parte Ayala, Binfa, Vanderstraeten y Bracke $^{(13)}$ estudian las identidades de enfermeras y parteras desde una perspectiva históricay sociológica. Para ello abordan las últimas cuatro décadas, donde ambas profesiones sanitarias son símiles y luego ambas emprenden trayectorias significativamente separadas en el país, convirtiéndose en ocupaciones diferentes a través de una alteridad socialmente construida. Fue en su interacción social, como escenario de competencia, que se presentan en conflictos socio-históricos por áreas de desempeño profesional superpuestas, como el cuidado del recién nacido, o los proyectos destinados a aliviar la escasez de enfermeras y el excedente de parteras a lo largo del período. Los autores concluyen que debido a esta interacción, que las relaciona socialmente, no es posible escribir separadamente la historia de una sola ocupación respecto de la otra. Por ello, plantean tener en cuenta la posibilidad de fusionar las dos ocupaciones, como un camino crítico que permita comprender el poder político de las profesiones del cuidado, y así, en conjunto, progresivamente, realizar prácticas más coherentes e integradas.

Ahora bien, en la zona sur de Chile, las autoras Paredes y Rivas ${ }^{(14)}$ estudian a la enfermería, pues coinciden que la evolución histórica del ejercicio profesional de las enfermeras ha sido poco documentada. Abarcan las décadas entre 1940 y 1980, dado que durante estas épocas surge la industrialización $y$, por consiguiente, aumenta vertiginosamente la industria hospitalaria. Asimismo, esbozan como hitos la creación de la medicina preventiva, la cooperación de la OPS, el ingreso de la Fundación Rockefeller, la creación del Servicio Nacional de Salud, el terremoto del año 1960 y la Creación de la Escuela de Enfermería de la Universidad Austral en el sur de Chile, por la necesidad de incrementar los profesionales de enfermería para la zona y el país. Concluyen que todos estos acontecimientos fueron relevantes para el desarrollo de la enfermería hospitalaria en el sur de Chile, seguido por el posicionamiento de la mujer en la sociedad. De esto se desprende que la práctica y el saber en el campo de la salud están ligados a las transformaciones históricas y el ejercicio profesional recibe una fuerte influencia de los contextos sociales, políticos y económicos que le acompañan.

Otra investigación, realizada por Chuaqui, Bettancourt, Leal y Aguirre $^{(15)}$, coincide en la escasez de indagaciones de corte histórico, y por ello buscan comprender la evolución de la identidad profesional de la enfermería de Valparaíso. Para ello abarcan 3 momentos, 1933-1968, 1968-1990 y 1990-2010. En el primer momento se crea la formación de la Escuela de Enfermería Carlos Van Buren, en la que se describe la emergencia del sistema público de salud y de la enfermería, que 
transita desde una política asistencial y caritativa a cargo de la iglesia, a una política general de salud liderada por el Estado. Es así que crean las primeras escuelas en Chile con fuertes exigencias morales. Se dibuja a la enfermería como el servir a otros, con abnegada vocación, en donde, además, se observan a sí mismas como "la salvadora" en una realidad de salud sumergida en la carencia de este período. Es por ello que concluyen que las enfermeras de este período valoran en gran medida su práctica, la cual se caracteriza por la ayuda al otro y la claridad de su función, por cierto, subordinada al médico.

Finalmente, Huaquián, Siles y Velandia ${ }^{(16)}$ describieron el origen de las prácticas de la enfermería chilena, prestando especial atención a la influencia española en dicho proceso. Los antecedentes bibliográficos revelan que en Chile no se ha desarrollado una investigación histórica profunda de los orígenes de la profesión, pues si bien existen textos desde 1902, en ellos no se abordan los siglos XVI, XVII y XVIII que corresponden al periodo colonial. Estos escritos señalan la influencia de los cuidados religiosos de los españoles en territorio americano, destacando Inés de Suárez como la primera médica-enfermera en Chile, la fundación del primer hospital por Pedro de Valdivia en el siglo XVI y la llegada de los hermanos de la Orden de San Juan de Dios en el siglo XVII. Esta última permaneció en Chile brindando asistencia hospitalaria y de enfermería a través de los cuidados religiosos. Huaiquián et al. concluyen que la llegada de la Orden mejoró sustancialmente la organización de los cuidados de salud al interior de los hospitales, tanto en áreas de administración, higiene, alimentación y, por sobre todo, de asistencia espiritual.

\section{Elementos historiográficos en la producción científica de libros}

Siles, Oguisso, Fernández y Souza ${ }^{(17)}$ realizaron una recopilación sobre la historia de la enfermería iberoamericana, describiéndola de manera lineal. En el capítulo VIII se aborda la historia de la enfermería en Chile, cuya autora, Ibarra $^{(18)}$, describe que la formación de la disciplina de Enfermería evolucionó desde un entorno de carencia sociosanitaria que impactó en la generación de la responsabilidad social asumida por la enfermera desde sus inicios. La autora relaciona la existencia de hitos propios del país y del mundo entre los siglos XIX y XX, que incidieron en la generación de la pobreza en los habitantes de Chile. Tales hitos fueron: el debilitamiento del orden capitalista mundial en 1870, la guerra del Pacífico en 1879, el progresivo despojo del campesinado tras la conquista de la Araucanía en 1880, la guerra civil de 1881 y la Primera Guerra Mundial, sucesos todos que determinaron que en Chile existiera hambre, cesantía, prostitución, hacinamiento, insalubridad, explotación, abandono, criminalidad y una alta mortalidad infantil, que era la más alta del mundo. Por ello, la descripción de la enfermería se situó, primariamente, en la práctica del conocimiento empírico y en la vocación religiosa.

Para ilustrar mejor esta descripción, el relato muestra que en el siglo XIX los cuidados eran entregados por religiosas, por ende, era la Iglesia la que ejercía la misericordia social y el cuidado. Estos cuidados fueron dando un giro a la salud popular que se brindaba, desde un eje caritativo, más vinculado a la aristocracia oligárquica y la iglesia, a una salud social y colectiva, que provenía de las exigencias de la ciencia y la agrupación médica, que imponían que el país dejara atrás lo privado y la caridad. La enfermería, en este marco, consolidaba su responsabilidad social como una contribución del profesional al estado sanitario del país.

Ahora bien, el texto de Flores titulado "Historia de la enfermería en Chile: Síntesis de su evolución educacional"(19), describe la enfermería desde sus inicios como profesión hasta el año 1965. La autora destaca que la enfermería evolucionó en torno al apoyo de destacados médicos que trajeron el modelo inglés a Chile, modelo que tenía la concepción de una enfermería laica y femenina. Los resultados se plasmaron en enfermeras eficientes, responsables, que cumplían a cabalidad las instrucciones médicas, dando un sello de abnegación y cooperación "médica", sello que continuó por muchos años en el ejercicio profesional, pues la comunidad médica veía que la enfermería era un apoyo crucial en la atención de los enfermos. Así mismo, se describe que los cargos directivos de las escuelas de Enfermería siempre estuvieron a cargo de médicos hasta el año 1947, en régimen de internados, en los cuales se entregaba una fuerte formación moral.

De acuerdo al relato de Flores ${ }^{(19)}$, se concluye que en el transcurso de la profesionalización de enfermería, los planes de estudios se modificaron de acuerdo a las nuevas necesidades que cons- 
tituían la creación del Ministerio de Salud, con planteamientos nuevos de filosofías y políticas públicas de salud. Así, la profesión de enfermería se adaptó y asumió un rol de empoderamiento, tanto en el eje sanitario como en el hospitalario. Ejemplos de estas iniciativas fueron la organización del servicio de enfermería a nivel nacional, el surgimiento de oportunidades de perfeccionamiento con apoyo de fundaciones internacionales, estancias docentes en los Estados Unidos y Canadá y un desarrollo de trabajo mancomunado entre el gobierno y las organizaciones no gubernamentales de salud. Asimismo, durante esta época se delimitaron las funciones que debían cumplir las profesionales, es decir, su quehacer e identidad.

Por otra parte, Cubillos, Castellano y Camus ${ }^{(20)}$, en el texto "Historia de la Escuela de Enfermería Isidora Lyon Cousiño 1950-2000", reitera el carácter religioso de los inicios de la enfermería y afirman que "la actitud del cuidado, que es la esencia de la enfermería, es tan antigua como la humanidad" y que el proceso de profesionalización de la enfermería está influido por los adelantos científicos y tecnológicos en el área de la medicina y por ello se necesitó formar una/un profesional que se dedicara al cuidado de los enfermos con conocimientos científicos. Esto impulsó la consolidación de la enfermería como profesión en el país. El libro relata la evolución de las escuelas hasta llegar a la escuela Isidora Lyon Cousiño, que ha sido la Escuela de Enfermería de la Pontificia Universidad Católica de Chile. Se describe exhaustivamente su desarrollo, dando énfasis a los proyectos que dicha escuela generó y aportó a la salud pública, con la línea de educación en salud y autocuidado en la enfermería moderna.

De modo similar, Bettancourt, Lazcano, Monardes, Núñez y Peroni ${ }^{(21)}$ describen que, a propósito de la celebración de los 75 años de la Escuela de Enfermería de la Universidad de Valparaíso, se realiza un relato historiográfico que da cuenta del desarrollo de la Escuela de Enfermería en una ciudad patrimonio de la humanidad como es Valparaíso. La enfermería profesional descrita se encuentra claramente asociada a la creación de la Escuela desde la filantropía de don Carlos Van Buren, y de ésta hacía la incorporación, como Escuela, a la red de atención sanitaria y de educación de la enfermería de la época. Destaca en su realización, la división de los periodos en torno a su desarrollo curricular, en donde uno de los períodos, denominado Tercera Etapa 1959-1969, se caracterizó por la fusión de la enseñanza de materias médicas y de enfermería en una asignatura: Proceso de Enfermería.

El último texto llamado "Introducción a la Historia de la Enfermería en Chile" de Siujen Chiang $^{(22)}$ aborda la temática de la historia de la profesión con la organización de datos, como una investigación periodística que está sustentada en discursos de autoridades y notas de prensa. Por ello, las afirmaciones poseen características globales y relatos de hitos en torno de la profesionalización de la enfermería. La narración relata algunas inconsistencias de autores tales como Ibarra $^{(18)}$ y Flores $^{(19)}$, como también afirmaciones disciplinarias descontextualizadas del período historiográfico.

\section{DISCUSIÓN}

Para encontrar evidencias respecto a la enfermera o la enfermería chilena como sujeto historiográfico en la producción de conocimiento disciplinario, es necesario comprender la cosmovisión que tienen algunos autores internacionales respecto de la historia de la enfermería.

Para las autoras Martínez y Chamorro ${ }^{(23)}$, la evolución de la historia de la enfermería española ha sido dividida en cuatro etapas, que corresponden a los distintos periodos de la evolución sociocultural del mundo occidental: 1) Etapa doméstica del cuidado, correspondiente a la Prehistoria y al período de existencia de las civilizaciones antiguas; 2) Etapa vocacional del cuidado, que corresponde al cuidado desarrollado durante la Edad media; 3) Etapa técnica, que ocurrió durante la Edad Moderna y, finalmente, 4) Etapa profesional, correspondiente a la Edad Contemporánea.

De acuerdo a este argumento, es posible señalar que la historia de la enfermería chilena está mayoritariamente explorada desde el desarrollo de su profesionalización, la cual se inicia a comienzos del siglo XX, por la creciente necesidad de la población de poseer cuidados científicos y no solamente desde la beneficencia de las órdenes religiosas y de la misericordia de las elites de la sociedad. La única autora que discurre desde una enfermería como oficio es Huaquián et al. ${ }^{(16)}$, quien conjuga la asimilación de una cultura religiosa 
desde la corona española a la naciente república de Chile.

En ese mismo sentido, Siles ${ }^{(24)}$ refiere que la historia de la enfermería permite que la disciplina sostenga un lenguaje propio, el cual sufre cambios a lo largo de los períodos historiográficos. Es así que sugiere que la historia de la enfermería puede utilizarse como un almacén de la memoria empírica de los cuidados en salud, como un depósito de los fundamentos de la salud y de la enfermedad en las diferentes culturas, y sus implicancias, en la forma de interpretar los cuidados. Además, la historia de la enfermería, según el mismo autor, puede ser considerada como un instrumento para el estudio monográfico de los cuidados en saludenfermedad, en épocas y culturas determinadas. También como historia comparada, que permite el análisis sistemático de las diferencias y semejanzas en procedimientos y en paradigmas interpretativos. Finalmente concluye que también es un instrumento para la transmisión de valores y actitudes hacia el cuidado de las personas.

Según lo anterior, es posible señalar que la historia de la enfermería escrita en los artículos y libros explorados constituye un almacén de memoria empírica respecto a la identidad de las enfermeras chilenas y de sus controversias durante la época del siglo XX, y así queda de manifiesto en los artículos de Ayala y Núñez ${ }^{(11)}$, de Núñez, Urra y Pavez ${ }^{(12)}$ y de Ayala et al. ${ }^{(13)}$. Asimismo, la realización de libros sobre Historia de Escuelas de Enfermería de la Pontificia Universidad Católica de Chile y de la Universidad de Valparaíso son la expresión de la historia como un instrumento de transmisión de valores y actitudes del cuidado con la impronta de dichas instituciones, la primera al mundo católico y la segunda vinculada al mundo laico de la profesión enfermería.

Por otra parte, Lewenson y McAllister ${ }^{(25)}$ demarcan que la historia de la enfermería comienza en los inicios del siglo XX, como una necesidad de las enfermeras en transmitir el conocimiento de la disciplina en las Escuelas de Enfermería. Sin embargo, durante la década de los años 1970, se constata que los cursos de historia de la enfermería se habían retirado progresivamente de los currículos de enfermería. Las autoras consideran que la resistencia a la historia de la enfermería se debía a la falta de conocimiento en el propio método historiográfico y que había una falta de rigor científico en su elaboración. Aparentemente, este último desafío permitió la creación en 1978 de la Asociación Americana de Historia de la Enfermería.

Ahora bien, Lewenson y McAllister ${ }^{(25)}$ revelan que la historia otorga conocimiento sobre cómo la profesión ha transitado en el contexto de los sistemas sanitarios, y cómo los avances de la medicina han influido en la transformación del rol de la enfermera, y cómo la relación del género, la raza y la etnicidad están intersectados con la economía y las políticas de cuidado. Por ello, resulta de gran interés conocer las historias de la enfermería de otros países, porque ese conocimiento permite contrastar las políticas de cuidado y los efectos del nacionalismo, del colonialismo y de las guerras.

Considerando lo anterior se comprende que Paredes y Rivas ${ }^{(14)}$, al situar su período historiográfico en 40 años de evolución, pudieron resaltar hitos sociales, políticos y económicos de Chile, los cuales influyeron en el desarrollo profesional de la enfermera hospitalaria de la zona sur del país. Por otra parte, Ibarra ${ }^{(18)}$ señala que las condiciones de carencias sociales de la población hasta mediados del siglo XX impactaron en la formación profesional de la enfermera. Asimismo, Flores ${ }^{(19)}$ menciona que el contexto de cambios en las temáticas sanitarias a nivel mundial permitieron que las enfermeras constituyeran sistemas de institucionalidad propios, que le permitieron empoderarse de su profesión.

Con los hallazgos encontrados en la presente revisión, es posible configurar que la enfermería, y su historia en Chile, es una temática incipiente y que se ha desarrollado en la comprensión de la identidad de las enfermeras y de sus vínculos con la institucionalidad de los sistemas sanitarios y educativos del país. Asimismo, se observa un mayor predominio del desarrollo de la historia de la enfermería vinculada al desarrollo profesional de la enfermería en el siglo XX, en detrimento del desarrollo de la enfermería comprendida como una disciplina. El único espacio de diferencia lo constituye el trabajo desarrollado por Huaiquián et al. ${ }^{(16)}$, quien realiza el trabajo de la enfermería desde la congregación San Juan de Dios, en el período historiográfico de la Colonia chilena.

Es importante clarificar que la historia de las enfermeras está tamizada, además, por las corrientes que las/los autoras/es adscriben para relatarla, y desde este pensamiento o cosmovisión, es posible sostener una coincidencia en la mayoría 
de los autores: la enfermera chilena es un sujeto historiográfico, porque es un grupo que al interior del ámbito de la salud posee el rol social de la continuidad e implementación de políticas sociosanitarias de un país. Por ello, su actuación frente a escenarios de crisis política y económica del país permite el avance o detención de la implementación de las políticas sanitarias.

Comprendiendo lo anterior, es posible señalar que estudiar a las enfermeras chilenas como grupo profesional, eminentemente femenino, ya consolidado en la mitad del siglo XX, permite comprender que luchaban por obtener un prestigio y un estatus al interior de la vida social y política de Chile, a diferencia de otros países que aún estaban situados en la fase de la enfermería como un oficio.

Es también clarificador entender que las enfermeras habían logrado su avance gracias a la comprensión e implementación de políticas de bienestar dirigidas al pueblo chileno por el Estado, lo que les permitió empoderarse en espacios sanitarios como la salud materno-infantil y comunitaria, y por ello gozaban de la creación de instituciones de enfermería, que también eran únicas en Latinoamérica.

\section{REFERENCIAS}

1. Velandia MA. Historia de la enfermería en Colombia. $1^{\text {a }}$ ed. Bogotá, Colombia: Ediciones de la Universidad Nacional de Colombia; 1995. 200 p.

2. Fawcett J. Tendencias de Investigación en Enfermería. Aquichán. 2014; 14(3): 289-93.

3. Durán DVMM. Una mirada hacia afuera: proyección de enfermería para el 2050. Aquichán [Internet]. 2018 Ene [citado 26 Ago 2019]; 18(1): 5-8. Disponible en: http://www.scielo. org.co/scielo.php?script=sci_arttext\&pid=S165759972018000100005\&lng=es

4. Chinn L, Kramer M. Knowlegde development in nursing theory and process. $10^{\mathrm{a}}$ ed. Estados Unidos: Elsevier; 2017. 301 p.

5. Lewis J. The Landscape of history. How Historians map the past. $1^{\mathrm{a}} \mathrm{ed}$. New York: Oxford University Press; 2002. 195 p.

6. Lewenson S, Kron E. Capturing Nursing History. $1^{\text {a }}$ ed. New York: Springer Publishing Company;

\section{CONCLUSIONES}

Para dar respuesta a la pregunta de investigación es posible afirmar que sus actores, es decir quienes están al interior de la disciplina, aún están en una etapa inicial respecto a valorar a la enfermera o a la enfermería como un sujeto o un espacio de discusión historiográfica.

En otras latitudes, esta pregunta ya tiene respuestas hace varias décadas y comparativamente, es evidente que la historiografía de la enfermería chilena es un espacio en construcción. Por ello, su tendencia a problematizar a la profesión de la enfermera más que al constructo disciplinario, es un campo a investigar.

Los desafíos que se proponen son: la narración de la enfermería en torno a la generación de un conocimiento cimentado en un proceso dialéctico de quien ofrece el cuidado y de quien lo recibe; la comprensión de la enfermera en la transformación de la salud de las clases populares del siglo XX; la permanente tensión al interior de los servicios sanitarios por las limitaciones de su autorregulación; o incluso, la transformación de la ciencia de enfermería, que está situada en la subsistencia del cuidado de las personas y las comunidades.

\section{8. $235 \mathrm{p}$.}

7. Association for the History of Nursing. Position paper on history in curriculum [Internet]. Mullica Hill (NJ): AAHN; 2001 Sept 21 [citado 28 Oct 2017]. Disponible en: https://www.aahn.org/ position-paper-on-history-in-curriculum

8. República de Chile. Censo de población de la República de Chile: Levantado el 15 de diciembre 1920 [Internet]. Dirección Nacional de Estadística. Santiago de Chile; 1925. 609 p. Colección Biblioteca Nacional de Chile [citado 28 oct 2017]. Disponible en: http://www.memoriachilena.gob. $\mathrm{cl} / 602 / \mathrm{w} 3$-article-82449.html

9. Mayers C. Valor social de la enfermera. Beneficencia [Internet]. 1929 Sept [citado 28 oct 2017]; 1(8): 425-6. Disponible en: http:// www.memoriachilena.gob.cl/archivos $2 / \mathrm{pdfs} /$ MC0065711.pdf

10. Salazar G. La Historia desde abajo y desde adentro. $1^{\text {a }}$ ed. Santiago de Chile: Taurus; 2017. 470 p.

11. Ayala R, Núñez E. Dusting of thelooking-glass: A historical analysis of the development of a 
nursing identity in Chile. Nurs Inq [Internet]. 2017 Ene 22 [citado 20 sept 2017]; 24(3). Disponible en: https://onlinelibrary.wiley.com/ doi/epdf/10.1111/nin.12185

12 Núñez E, Urra E, Pavez A. Identidad e institucionalidad de las enfermeras chilenas en la mitad del siglo XX. Cienc enferm [Internet]. 2016 abr [citado 20 sept 2017]; 22(1): 135-45. Disponible en: https://scielo.conicyt.cl/pdf/cienf/ v22n1/art_12.pdf

13. AyalaR,BinfaL,VanderstraetenR,BrackeP.Exploring historical conflicts between midwives and nurses: A perspective from Chile. J Interprof Care [Internet]. 2014 sept [citado 20 sept 2017]; 29 (3): 216-22. Disponible en: https:/www.tandfonline.com/ doi/pdf/10.3109/13561820.2014.956863?casa_ token=-SbDRC-jpN4AAAAA:kY_YRLp4e2L-E5 W71UwpLzHEGao41189ndYdqhau9MTiK_ JckFrBrwpCdCBW4MS3fduxlT3haIBQtfI

14. Paredes P, Rivas E. Historia del ejercicio profesional de enfermeras hospitalarias del Sur de Chile (19401980). Cienc enferm [Internet]. 2014 abr [citado 8 oct 2017]; 20(1): 9-21. Disponible en: https:// scielo.conicyt.cl/pdf/cienf/v20n1/art_02.pdf

15. Chuaqui-Kettlun J, Bettancourt-Ortega L, LealRomán V, Aguirre-González C. La identidad profesional de la enfermería: un análisis cualitativo de la enfermería en Valparaíso (1933-2010). Aquichán [Internet]. 2014 mar [citado 08 oct 2017]; 14(1): 53-66. Disponible en: http:// www.scielo.org.co/scialo.php?pid =S1657$59972014000100006 \&$ script =sci abstract\&tlng=es

16. Huaquián-Silva J, Siles-González J, VelandiaMora A. La enfermería de la Orden de San Juan de Dios en el Chile colonial. Aquichán [Internet]. 2013 ago [citado 08 oct 2017]; 13(2): 290-300. Disponible en: http://www.scielo.org. co/scielo.php?script=sci_arttext\&pid=S165759972013000200015\&lng=es

17. Siles J, Oguisso T, Fernandes G, De Souza P.
Cultura de los cuidados: Historia de la enfermería iberoamericana. Alicante, España: ECU; 2010. $420 \mathrm{p}$.

18. Ibarra MX. Historia de la Enfermería en Chile. En: González Siles J, Oguisso T, Fernandes de Freitas G. Cultura de los cuidados: historia de la enfermería iberoamericana. Alicante: ECU; 2010. p. 265-289.

19. Flores R. Historia de la enfermería en Chile, Síntesis de su evolución educacional. Santiago, Chile: Memoria Chilena; 1965. 147 p.

20. Cubillos O, Castellano S, Camus G. Historia Escuela de Enfermería "Isidora Lyon Cousiño 1950-2000. Santiago, Chile: Centro de Documentación e Investigaciones Históricas de la Facultad de Medicina UC; 2000. 310 p.

21. Bettancourt L, Lazcano M, Monardes G, Núñez N, Peroni S. Escuela de Enfermería: 75 ańos formando profesionales de enfermería al cuidado de la comunidad. Valparaíso, Chile: Escuela de Enfermería, Facultad de Medicina, Universidad de Valparaíso; 2010.

22. Chiang S. Introducción a la Historia de la Enfermería en Chile. Santiago, Chile: Ediciones Técnicas de Educación Superior; 2010. 185 p.

23. Martínez M, Chamorro E. Historia de la Enfermería. Evolución histórica del cuidado enfermero. $2^{a}$ ed. Madrid, España: ELSEVIER; 2011. $160 \mathrm{p}$.

24. Siles-González J. Historia de la Enfermería. 1ª ed. España: Aguaclara; 2008. 375 p.

25. Lewenson SB, McAllister A. Cap 1. Learning the Historical Method: Step by Step. En: De Chesnay M, editor. Nursing Research using Historical Methods: Qualitative Designs and Methods in Nursing [Internet]. New York (US): Springer Publishing Company; 2015 [citado 08 oct 2017]. p. 1-21. Disponible en: http://ghttp.48653. nexcesscdn.net/80223CF/springer-static/media/ samplechapters/9780826126177/9780826126177_ chapter.pdf 